\title{
Balanced Crystalloid Solution Versus Normal Saline on Biochemical Outcomes in Acute Medical and Surgical Patients
}

\author{
Liang Qu ${ }^{\mathrm{a}}$, Evan Newnham ${ }^{\mathrm{b}}$, Salena Ward ${ }^{\mathrm{c}}$, Louis Huang ${ }^{\mathrm{a}, \mathrm{b}}$, Robert MacGinley ${ }^{\mathrm{a}, \mathrm{b}, \mathrm{d}}$, \\ Lawrence P McMahon ${ }^{\mathrm{b}}$
}

\begin{abstract}
Background: Concerns have been raised regarding the use of various intravenous fluids resulting in hyperchloremic acidosis and associated acute kidney injury; however, this has primarily been in the context of resuscitation and critical care, and not ward-based medical or surgical patients who receive lower volumes of infusion fluid. We aimed to determine whether normal saline use is more likely to produce hyperchloremic metabolic acidosis than compound sodium lactate, in acute general medical and surgical patients.
\end{abstract}

Methods: This was a retrospective study, conducted in 2015 in a metropolitan hospital in Melbourne, Australia, and of 1,158 sequentially admitted general medical and surgical patients receiving either intravenous normal saline or compound sodium lactate. Demographics, admission diagnosis, comorbidities, and medications were compared. Serum biochemistry results $24-48$ h post-infusion were analyzed. Primary outcome was incidence of serum hyperchloremia post-infusion. In addition, mean shift in serum chloride concentration as well as mean normalized chloride concentration $(\mathrm{mmol} / \mathrm{L}$ per day per $\mathrm{L}$ of infusion) were calculated. Acute kidney injuries and deaths during admission were also reported.

Results: A total of 221 patients (169 medical and 52 surgical patients) were included for analysis (mean age 71.6 years, $72 \%$ female), with 174 normal saline and 61 compound sodium lactate infusions. No difference in hyperchloremia rate was found (normal saline vs. compound sodium lactate; $\mathrm{n}=8(4.6 \%)$ vs. $\mathrm{n}=2(3.3 \%), \mathrm{P}=1.0)$. Post-infusion serum chloride concentration was also not significantly different $(99.0 \pm 5.1 \mathrm{mmol} / \mathrm{L}$ vs. $100.0 \pm 3.4 \mathrm{mmol} / \mathrm{L}, \mathrm{P}=0.1700)$ between infusion groups. Normal saline infusions resulted in an increase of mean normalized chloride concentration compared to compound sodium lactate $(1.2 \pm 4.0 \mathrm{mmol} / \mathrm{L}$ per day/L vs. $-0.3 \pm 1.5, \mathrm{P}$

Manuscript submitted March 8, 2018, accepted March 29, 2018

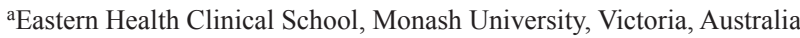
${ }^{b}$ Department of Renal Medicine, Eastern Health, Victoria, Australia 'Department of Surgery, Eastern Health, Melbourne, Victoria, Australia ${ }^{\mathrm{d} C}$ Corresponding Author: Robert MacGinley, Eastern Health Clinical School, Monash University, Level 2, 5 Arnold Street, Box Hill VIC 3128, Australia. Email: Robert.macginley@monash.edu

doi: https://doi.org/10.14740/wjnu339w
$=0.0121)$

Conclusions: We conclude that 2 - 3 L normal saline infusions in this population do not appear to be associated with hyperchloremic acidosis more frequently than compound sodium lactate infusions, allaying concerns of normal saline use in wards.

Keywords: Intravenous fluid therapy; Acute kidney injury; Hyperchloremic metabolic acidosis; Normal saline; Compound sodium lactate

\section{Introduction}

Extensive literature has been published regarding the association between large volume intravenous normal saline (NS) infusions and hyperchloremic metabolic acidosis (HCMA). Well-established theories have been put forth explaining the process of saline-induced acidosis, including the proposal that the increase in chloride ion concentration and the dilution of bicarbonate results in systemic acidosis $[1,2]$.

The reported clinical effects of these large volume NS infusions however, are varied and often contrasting but concerning enough to limit NS use. NS use has been shown to lead to renal vasoconstriction, delayed micturition, increased acute kidney injury (AKI), and hyperkalemia [3, 4]. The resultant HCMA has also been shown to be pro-inflammatory [5]. This is opposed to other studies, which have shown that HCMA is not associated with AKI, the high chloride concentration presumed to be a benign effect of large volume infusions [6].

Much of the above discussion has currently been in the critical care setting or in resuscitation, where determining the presence of HCMA can be crucial for guiding further management [7]. It is not known whether the concerns for HCMA are equally relevant in a general medical or surgical unit, with a different patient population. The investigation of non-critically ill patients has been recently reported in a large-scale randomized trial, however the outcomes measured in this study were primarily clinical outcomes: hospital-free days and adverse kidney events like death, renal-replacement therapy and persistent renal dysfunction [8]. Our study focuses on the intermediate biochemical measure of hyperchloremia, while 


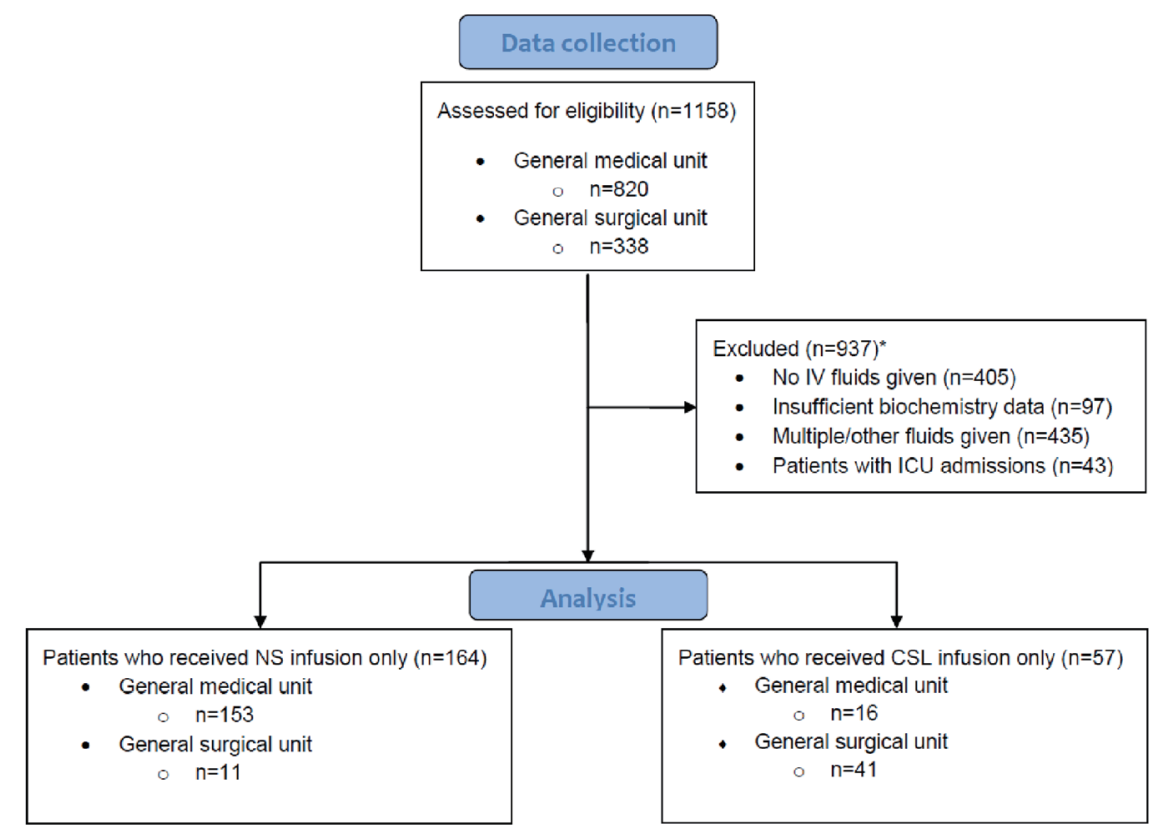

Figure 1. Criteria used for exclusion to obtain list of patients for final analysis. *Number of patients satisfying each exclusion criteria overlapped with other criteria. In total, there were 937 patients excluded from this study. CSL: compound sodium lactate; ICU: intensive care unit; IV: intravenous; NS: normal saline.

also further exploring the effects of infusing NS with lower volumes (typically volumes less than $3 \mathrm{~L}$ per $24 \mathrm{~h}$ ), than in the critically ill population $[1,2]$.

Our study therefore aimed to investigate any significant biochemical effects of maintenance intravenous NS infusions compared to a buffered crystalloid solution, compound sodium lactate (CSL), in acute general medical and surgical patients with a variety of diagnoses.

\section{Methods}

We performed a retrospective single-centre cohort study conducted of inpatients at a secondary care hospital in metropolitan Melbourne, Australia. Data were collected from sequentially admitted patients from a general medical and a general surgical unit. These patients were sequentially admitted between May to October 2015. This study was approved through the Eastern Health Human Research Ethics Committee.

The electronic records accessed included laboratory results and scanned medical records. Relevant data were obtained from discharge summaries, medication charts, and fluid infusion charts. Data included patient demographics, comorbidities, admission length and reason, medications administered, and intravenous fluid infusions administered during the admission. Any intensive care admissions were noted for patients in this study. Comorbidity data were collected for any chronic cardiovascular, renal, or respiratory disease. Any medication data were also collected for patients, for angiotensin converting enzyme (ACE) inhibitors, angiotensin II receptor blockers (ARBs), non-steroidal anti-inflammatory drugs (NSAIDs), and diuretics.
Patients were excluded from this study depending on the suitability of their fluid or biochemistry data (Fig. 1). The exclusion criteria included: not having received any fluids during the course of the admission, receiving multiple types of fluids within their infusion course or receiving other fluids not relevant to this study, and also if there were insufficient biochemistry results to allow for analysis. Patients were also excluded from this study if they were admitted to intensive care during the course of their hospital admission.

For the purpose of this study, infusions of NS only or CSL only were considered for analysis. Any intravenous therapy that the patient received was recorded based on the number of days into admission the fluid was given, the volume, as well as the type of fluid given. The biochemistry data were collected 24 - $48 \mathrm{~h}$ post-infusion therapy. A baseline set of biochemistry data, typically pre-infusion (e.g. at admission) were also obtained. The serum biochemistry results collected were: sodium, chloride, bicarbonate, potassium, creatinine, and estimated glomerular filtration rate (eGFR). The measure of hyperchloremia, defined as $>105 \mathrm{mmol} / \mathrm{L}$, was used to indirectly indicate HCMA, as laboratory data on $\mathrm{pH}$ was not collected.

The proportion of patients who became newly hyperchloremic post-infusion was compared between NS and CSL groups. In addition, mean post-infusion serum electrolyte concentrations were compared between patients of the two infusion types. To further explore the effects of fluid administration on serum chloride levels, a normalized chloride concentration ratio was reported. This was calculated through the difference in chloride concentration before and after each infusion, and accounting for time (days) as well as volume (L). The value reported was the mean change in chloride concentration per day, per $\mathrm{L}$ of infusion $\left(\Delta \mathrm{Cl}^{-} /\right.$day/L). 
Table 1. Characteristics of Patients Who Received Intravenous Fluids During Their Admission

\begin{tabular}{|c|c|c|c|c|}
\hline \multirow{2}{*}{ Demographics } & \multicolumn{2}{|c|}{ Fluid infusion given } & \multirow{2}{*}{ All patients $(n=221)$} & \multirow{2}{*}{ P-value } \\
\hline & NS $(n=164)$ & $\operatorname{CSL}(n=57)$ & & \\
\hline Age, mean (SD), year & $76.4(17.5)$ & $57.9(21.6)$ & $71.6(20.3)$ & $<0.0001$ \\
\hline \multicolumn{5}{|l|}{ Admitting unit } \\
\hline Medical & $153(93.3 \%)$ & $16(28.1 \%)$ & $169(76.5 \%)$ & $<0.0001$ \\
\hline \multicolumn{5}{|l|}{ Comorbidities } \\
\hline Hypertension & $79(48.2 \%)$ & $18(31.6 \%)$ & $97(43.9 \%)$ & 0.0297 \\
\hline Ischemic heart disease & $32(19.5 \%)$ & $5(8.8 \%)$ & $37(16.7 \%)$ & 0.0614 \\
\hline Diabetes mellitus & $46(28.0 \%)$ & $12(21.1 \%)$ & $58(26.2 \%)$ & 0.3010 \\
\hline Chronic respiratory disease & $30(18.3 \%)$ & $9(15.8 \%)$ & $39(17.6 \%)$ & 0.6693 \\
\hline Loop diuretics & $56(34.1 \%)$ & $9(15.8 \%)$ & $65(29.4 \%)$ & 0.0088 \\
\hline Thiazide diuretics & $9(5.5 \%)$ & $4(7.0 \%)$ & $13(5.9 \%)$ & 0.6724 \\
\hline Potassium sparing diuretics & $11(6.7 \%)$ & $3(1.8 \%)$ & $12(5.4 \%)$ & 0.6998 \\
\hline
\end{tabular}

SD: standard deviation; ACEI: ACE inhibitor; ARB: angiotensin II receptor blocker; NSAID: non-steroidal anti-inflammatory drug. The specific ACEls (ramipril, perindopril, fosinopril, enalapril), ARBs (candesartan, irbesartan, telmisartan, olmesartan), and NSAIDs (aspirin, ibuprofen, celecoxib, diclofenac) were also recorded.

For additional characterisation of the patients in this study, the number of individuals who had AKI during their admission was also recorded from the biochemistry data. For this study, AKI was defined based on the Kidney Disease: Improving Global Outcomes (KDIGO) criteria of an increase in serum creatinine of $>26.5 \mathrm{mmol} / \mathrm{L}$ or $>1.5 \times$ increase from baseline serum creatinine within $48 \mathrm{~h}$, during the course of their admission [9].

\section{Statistical analysis}

The mean volume of infusion was calculated for the number of infusion courses given across all inpatients. Data were analyzed comparing the NS and CSL cohorts, for all patients pooled together from both medical and surgical units. A further subgroup analysis was also completed for both medical patients and surgical patients. The primary analysis was to determine any significant difference in the proportion of patients developing hyperchloremia post-infusion in the NS and CSL groups, using Fisher's exact test. Means and standard deviations were also calculated for all measured serum electrolyte values post-infusion between the NS and CSL groups. A twosided $t$-test was used to compare mean post-infusion electrolyte values between the two infusion types, and statistical significance was reported using P-values. An $\alpha$-value of $\mathrm{P}<0.05$ was used to deem significance. The mean and standard deviation of the normalized chloride concentrations were calculated for the two infusion groups. A two-sided $t$-test was used to identify any difference in the change of serum chloride concentration normalized to time and volume. Multivariate analysis of the data was considered initially but was omitted due to the small sample size.

A secondary analysis regarding fluid therapy prescribing differences between the medical and surgical units was also calculated, using Fisher's test calculations. This was used to determine whether there was an association with a particular type of fluid prescribed as single fluid therapy, in relation to a particular hospital unit.

\section{Results}

Data were collected from a total of 820 general medical and 338 general surgical inpatients. A total of 937 patients were excluded from this study (Fig. 1), leaving 169 medical and 52 surgical patients for analysis. Comparing baseline characteristics of the NS (164 patients) and CSL (57 patients) cohorts, there was a significant difference in the mean age $(\mathrm{P}<0.0001)$, as well as the proportion of medical versus surgical units prescribing the fluid. Other demographic comparators, including gender and comorbidities, were mostly similar between the two groups (Table 1).

From the 221 patients included for analysis, there were 235 fluid infusion data sets (174 NS infusions and 61 CSL infusions). The medical group was given $161 \mathrm{NS}$ and $17 \mathrm{CSL}$ infusions, while the surgical group was given $13 \mathrm{NS}$ and $44 \mathrm{CSL}$ infusions. The mean volume of infusion was $1.8 \pm 1.3 \mathrm{~L}$ and 
Serum Electolytes in Pooled Medical \& Surgical Patients

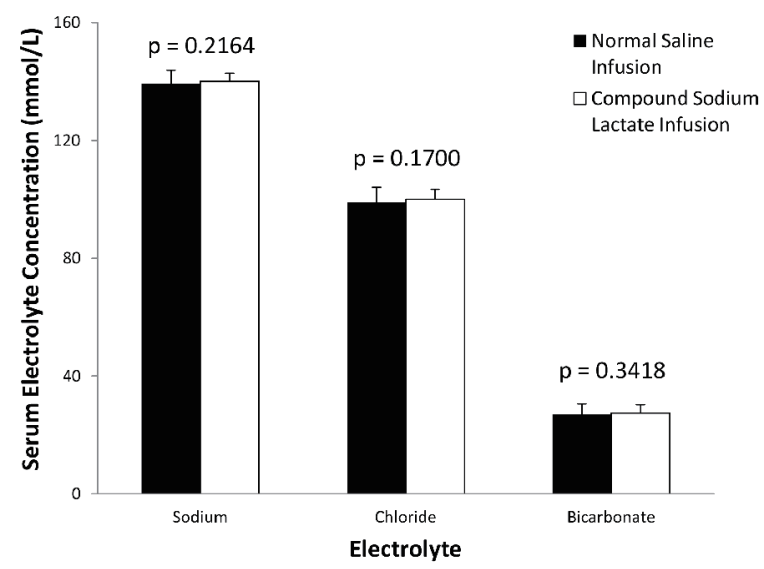

Figure 2. Post-infusion mean values of electrolytes between NS and CSL cohorts. There were no significant differences in the mean values of serum sodium, chloride and bicarbonate post-infusion of both normal saline and compound sodium lactate.

$2.6 \pm 1.6 \mathrm{~L}$ for the NS and CSL cohorts respectively. The mean volume of infusion for all patients combined was $2.0 \pm 1.4 \mathrm{~L}$.

\section{Differences in serum biochemistry}

Of the 174 NS infusions analyzed, six infusions led to the development of hyperchloremia that was not present at baseline (4.6\%). Of the $61 \mathrm{CSL}$ infusions analyzed, two infusions led to the development of hyperchloremia $(3.3 \%)$. There was no significant difference in the proportion of infusions that led to the development of hyperchloremia between the NS and CSL groups $(\mathrm{P}=1.000)$.

There were no significant differences in post-infusion mean serum electrolyte values between NS and CSL infused patients (Fig. 2). There was no difference in serum sodium (NS: $139.3 \pm$ $4.6 \mathrm{mmol} / \mathrm{L}$ vs. CSL: $140.1 \pm 2.7 \mathrm{mmol} / \mathrm{L}, \mathrm{P}=0.2164)$, chloride $(99.0 \pm 5.1 \mathrm{mmol} / \mathrm{L}$ vs. $100.0 \pm 3.4 \mathrm{mmol} / \mathrm{L}, \mathrm{P}=0.1700)$, or bicarbonate levels $(26.9 \pm 3.6 \mathrm{mmol} / \mathrm{L}$ vs. $27.4 \pm 2.8 \mathrm{mmol} / \mathrm{L}$, $\mathrm{P}=0.3418)$. No significant differences were also observed in subgroup analysis of general medical and surgical patients (Table 2). In the subgroup of general medical patients, serum bicarbonate $(26.9 \pm 3.6 \mathrm{mmol} / \mathrm{L}$ vs. $27.0 \pm 3.6 \mathrm{mmol} / \mathrm{L}, \mathrm{P}=$ $0.9159)$ or chloride $(98.9 \pm 5.2 \mathrm{mmol} / \mathrm{L}$ vs. $99.4 \pm 5.5 \mathrm{mmol} / \mathrm{L}$, $\mathrm{P}=0.7163)$ in those treated with NS and CSL respectively,
Table 2. Post-Infusion Mean Serum Electrolyte Values Comparing Normal Saline and Compound Sodium Lactate Infused Patients

\begin{tabular}{|llll}
\hline \multirow{2}{*}{ Biochemistry } & \multicolumn{3}{c}{ Concentration $(\mathrm{mmol} / \mathrm{L}) \pm$ SD } \\
\cline { 2 - 4 } & $\mathrm{NS}$ & CSL & P-value \\
\hline Medical & $(\mathrm{n}=153)$ & $(\mathrm{n}=16)$ & \\
$\quad$ Serum sodium & $139.2 \pm 4.7$ & $140.0 \pm 4.0$ & 0.5127 \\
Serum chloride & $98.9 \pm 5.2$ & $99.4 \pm 5.5$ & 0.7163 \\
$\quad$ Serum bicarbonate & $26.9 \pm 3.6$ & $27.0 \pm 3.6$ & 0.9159 \\
Surgical & $(\mathrm{n}=11)$ & $(\mathrm{n}=41)$ & \\
\hline Serum sodium & $140.9 \pm 3.7$ & $140.1 \pm 2.1$ & 0.3511 \\
Serum chloride & $100.6 \pm 3.4$ & $100.2 \pm 2.2$ & 0.6378 \\
Serum bicarbonate & $26.7 \pm 3.3$ & $27.5 \pm 2.5$ & 0.3834 \\
All patients & $(\mathrm{n}=164)$ & $(\mathrm{n}=57)$ & \\
Serum sodium & $139.3 \pm 4.6$ & $140.1 \pm 2.7$ & 0.2164 \\
Serum chloride & $99.0 \pm 5.1$ & $100.0 \pm 3.4$ & 0.1700 \\
Serum bicarbonate & $26.9 \pm 3.6$ & $27.4 \pm 2.8$ & 0.3418 \\
\hline
\end{tabular}

CSL: compound sodium lactate; NS: normal saline; SD: standard deviation.

were not significantly different. The results in the surgical subgroup including bicarbonate $(26.7 \pm 3.3 \mathrm{mmol} / \mathrm{L}$ vs. $27.5 \pm 2.5$ $\mathrm{mmol} / \mathrm{L}, \mathrm{P}=0.3834)$ or chloride $(100.6 \pm 5.2 \mathrm{mmol} / \mathrm{L}$ vs. 100.2 $\pm 2.2 \mathrm{mmol} / \mathrm{L}, \mathrm{P}=0.6378$ ) in those treated with NS and CSL respectively were also not significantly different.

The normalized serum chloride concentration change was calculated for the NS and CSL infusion groups. For patients who received NS infusions, the mean serum $\Delta \mathrm{Cl}^{-} /$day/L was calculated to be an increase of $1.2 \pm 4.0 \mathrm{mmol} / \mathrm{L}$ per day per $\mathrm{L}$ of infusion. Patients who received CSL infusions had a mean decrease of $0.3 \pm 1.5 \mathrm{mmol} / \mathrm{L}$ per day per L of infusion. A statistical significance was noted between the NS and CSL infusion groups $(95 \% \mathrm{CI} 0.35-2.82, \mathrm{P}=0.0121)$.

\section{Fluid infusion and patient outcomes}

Patients who received NS infusions were more likely to have a longer hospital stay (Table 3). The mean length of admission was 5.8 days for patients who received NS infusions, compared to 3.5 days for patients who had CSL infusions $(\mathrm{P}<0.0007)$. Of the patients who received NS infusions, there were also 10 patients $(6.1 \%)$ who experienced AKI (according to biochemi-

Table 3. Summary Statistics of Patient Outcomes Broken Down by Fluid Infusion Received

\begin{tabular}{llll}
\hline \multirow{2}{*}{ Outcomes } & \multicolumn{3}{c}{ No. } \\
\cline { 2 - 4 } & NS $(\mathbf{n}=\mathbf{1 6 4})$ & CSL $(\mathbf{n}=\mathbf{5 7})$ & P-value \\
\hline Length of stay, mean (SD), days & $5.8(4.9)$ & $3.5(2.2)$ & 0.0007 \\
Acute kidney injury (KDIGO) & $10(6.1 \%)$ & $0(0.0 \%)$ & 0.0564 \\
Death during admission & $6(3.7 \%)$ & $0(0.0 \%)$ & 0.1432 \\
\hline
\end{tabular}

KDIGO: Kidney Disease: Improving Global Outcomes clinical practice guideline 2009. 
cal criteria from KDIGO) during their admission, and six patients $(3.7 \%)$ who died during admission. There were no cases of AKI or deaths, from the CSL cohort. These results for AKI and deaths during admission were however not statistically significant, hence no associations were found for a particular fluid infusion.

\section{Fluid prescribing differences between units}

There was a significant difference in the choice of prescribing fluids between the general medical unit and the general surgical unit. The medical unit was associated with more prescriptions of NS infusions, where $161 \mathrm{NS}$ and $17 \mathrm{CSL}$ infusions were given. Meanwhile, the surgical group was more likely to prescribe CSL infusions, where $13 \mathrm{NS}$ and $44 \mathrm{CSL}$ infusions were given $(\mathrm{P}<0.0001)$.

\section{Discussion}

This study aimed to provide an insight into how fluid prescribing practices in general medical and surgical units affect the serum biochemistry of patients. In particular, we sought evidence for differences in the incidence of HCMA relating to the type of fluid infused. In our study, there was no significant increase in the development of hyperchloremia for patients who received NS infusions, compared to those receiving CSL infusions. The surrogate measure of hyperchloremia without measuring $\mathrm{pH}$ was used to indirectly infer HCMA. There was a low occurrence of hyperchloremia, comparable between both infusion groups, with a highly non-significant $\mathrm{P}$-value ( $\mathrm{P}$ $=1.0$ ). Although the study was limited by not being able to collect data on acidosis, these findings suggest that HCMA is a condition that would infrequently affect general medical and surgical patients given under $3 \mathrm{~L}$ of fluid. Furthermore, there was no significant difference in the post-infusion mean serum sodium, chloride or bicarbonate levels when comparing NS to CSL infusions for all patients, as well as in the medical and surgical subgroups. The normalized change in chloride concentration per day per L of infusion was calculated for both the patients who were infused with NS and those with CSL. There was a significant difference in the change in chloride concentration noted $(95 \%$ CI $0.34-2.82, \mathrm{P}=0.0121)$, however this change is clinically insignificant.

These findings are consistent with the recently published data from Self et al [8] in fluid administration for non-critically ill patients. Outcome measures used in their controlled trial were primarily clinical, although changes in serum biochemistry were also reported. In their study however, serum biochemistry changes were studied for patients upon presentation to the emergency department, the patient population hence differs to our general medical and surgical unit inpatients who receive intravenous therapy in lower rates. Nevertheless, our results strengthen the suspicion that there is no discernible difference in the use of NS compared to CSL infusions in outcomes relevant to kidney function, in non-critically ill patients. It is also worthy to note that our study represents the first Australian based cohort study in this field, extending the generalisability of the large-scale trial results demonstrated in the United States $[8,10]$.

In addition to the above biochemical findings, our secondary clinical outcome results demonstrated no significant difference for deaths during admission between NS and CSL infused groups. AKI rates between NS and CSL groups bordered significance $(\mathrm{P}=0.0564)$. The possibility of NS infusions leading to AKI from this study should be interpreted alongside other studies investigating the effect of NS infusions. NS infusions have been associated with greater chloride administration and high rates of resultant renal dysfunction in some cases $[1,11]$. On the contrary, recent evidences in both the critical and noncritical care setting (with both small and large volume infusions), have suggested that there is no significant difference in severe AKI rates when comparing NS to a balanced crystalloid solution $[2,7,8,10]$. Our data add to the literature investigating the effects of intravenous infusions on AKI incidence.

We believe this is the first study to investigate the presence of adverse effects of NS infusions in a general medical or surgical setting with volumes under $3 \mathrm{~L}$. Our study is strengthened by robust data collecting methods for fluids administered, along with objective and standardised biochemistry results for analysis. Nonetheless, there are also limitations relating mainly to the limits of collecting medical record data. In particular, the comorbidities taken from discharge summaries may be incomplete when compared to accessible investigation results (e.g. chronic kidney disease (eGFR $<60 \mathrm{~mL} / \mathrm{min}$ ) was not included in this study as a comorbidity, as this condition in the discharge summaries did not correspond when audited against the biochemical results that were available, i.e. a proportion of the patients were underreported). This study has also not explored the impact that concurrent medications have (we did not exclude the use of diuretics as an example). Data on oral intake were also not available. These parameters would be useful for a future prospective study as opposed to a retrospective collection in this study. Our data on fluid infusions were limited not only by the manual recording of volume infused, but also from not stating the volume status of the patient before and after, but also the reason why particular fluids were prescribed, masking potential indications such as correcting fluid imbalances. Unfortunately, due to the nature of retrospective studies like this, our sample population cohorts were also not completely similar in patient demographics potentially causing confounding by selection biases. Any imbalance in comorbidity prevalence, as well as absence of diagnosis information may also be a confounder in the study regards fluid choices. Finally the limitation of only studying patients with sufficient data available affects the overall impact of our findings. Multivariate analysis was omitted from this study, as additional strong inferences would not be likely drawn from our relatively small sample size. In addition, a choice to not implement a severity of illness score (such as a Charlson comorbidity index), was made due to the heterogeneous data that were obtained from medical records. The varying amount of information recorded would limit the application and utility of this additional analysis.

An unexpected positive result was the longer length of stay in patients receiving NS compared to CSL. Other previous studies on this topic have not observed this effect. We believe 
that confounders may have contributed to our positive result. Despite the cohorts exhibiting mostly similar baseline characteristics, there was a very significant difference in the proportion of patients from medical or surgical units in the NS and the CSL cohorts. It is likely that the length of stay is influenced by age as well as the reason for admission, related to the admitting unit for which the patients were categorised under. Depending on the diagnosis that the patient is admitted for, as well as the indication for the fluid being prescribed, this may account for the effects observed on length of stay, and in association, severity of illness. Future studies researching the use of crystalloid infusions in general medical and surgical patients may be able to expand on the findings seen here, by looking at the indications for fluids administered like dehydration.

\section{Conclusions}

Our study did not find an association with HCMA when volumes of under $3 \mathrm{~L}$ of fluid (NS compared to CSL) for general medical or surgical patients. This study is in contrast with previous studies of large volume use, where NS infusions have been suggested to result in adverse effects. This study will aid fluid prescription preferences when considering clinician's concern for adverse effects accompanying intravenous fluid therapy.

\section{Grant Support}

None.

\section{Financial Disclosures}

The authors confirm that they have no relevant financial disclosures.

\section{Conflict of Interest}

The authors declare that there is no relevant conflict of interest to declare.

\section{References}

1. Severs D, Hoorn EJ, Rookmaaker MB. A critical appraisal of intravenous fluids: from the physiological basis to clinical evidence. Nephrol Dial Transplant. 2015;30(2):178187.

2. Ince $\mathrm{C}$, Groeneveld $\mathrm{AB}$. The case for $0.9 \% \mathrm{NaCl}$ : is the undefendable, defensible? Kidney Int. 2014;86(6):10871095.

3. Moritz ML, Ayus JC. Maintenance intravenous fluids in acutely ill patients. N Engl J Med. 2015;373(14):13501360.

4. Chowdhury AH, Cox EF, Francis ST, Lobo DN. A randomized, controlled, double-blind crossover study on the effects of 2 -L infusions of $0.9 \%$ saline and plasmalyte(R) 148 on renal blood flow velocity and renal cortical tissue perfusion in healthy volunteers. Ann Surg. 2012;256(1):18-24.

5. Kellum JA, Song M, Almasri E. Hyperchloremic acidosis increases circulating inflammatory molecules in experimental sepsis. Chest. 2006;130(4):962-967.

6. Guidet B, Martinet O, Boulain T, Philippart F, Poussel JF, Maizel J, Forceville X, et al. Assessment of hemodynamic efficacy and safety of $6 \%$ hydroxyethylstarch 130/0.4 vs. $0.9 \% \mathrm{NaCl}$ fluid replacement in patients with severe sepsis: the CRYSTMAS study. Crit Care. 2012;16(3):R94.

7. Young P, Bailey M, Beasley R, Henderson S, Mackle D, McArthur C, McGuinness S, et al. Effect of a Buffered Crystalloid Solution vs Saline on Acute Kidney Injury Among Patients in the Intensive Care Unit: The SPLIT Randomized Clinical Trial. JAMA. 2015;314(16):17011710 .

8. Self WH, Semler MW, Wanderer JP, Wang L, Byrne DW, Collins SP, Slovis CM, et al. Balanced crystalloids versus saline in noncritically ill adults. N Engl J Med. 2018;378(9):819-828.

9. Kidney Disease: Improving Global Outcomes CKDMBDWG. KDIGO clinical practice guideline for the diagnosis, evaluation, prevention, and treatment of Chronic Kidney Disease-Mineral and Bone Disorder (CKD-MBD). Kidney Int Suppl. 2009;113:S1-130.

10. Semler MW, Self WH, Wanderer JP, Ehrenfeld JM, Wang L, Byrne DW, Stollings JL, et al. Balanced Crystalloids versus Saline in Critically Ill Adults. N Engl J Med. 2018;378(9):829-839.

11. Yunos NM, Bellomo R, Hegarty C, Story D, Ho L, Bailey M. Association between a chloride-liberal vs chloride-restrictive intravenous fluid administration strategy and kidney injury in critically ill adults. JAMA. 2012;308(15):1566-1572. 